\title{
Stereotactic or conventional radiotherapy for macroscopic prostate bed recurrence: a propensity score analysis
}

\author{
Giulio Francolini ${ }^{1}\left[\right.$ [D Barbara Alicja Jereczek-Fossa ${ }^{2,3} \cdot$ Vanessa Di Cataldo ${ }^{1,4} \cdot$ Gabriele Simontacchi $^{1}$. \\ Giulia Marvaso $^{2,3} \cdot$ Sara Gandini $^{5} \cdot$ Federica Corso $^{5} \cdot$ Lucia Pia Ciccone $^{6} \cdot$ Maria Alessia Zerella ${ }^{2,3} \cdot$ Piercarlo Gentile $^{7}$. \\ Federico Bianciardi ${ }^{7}$. Sara Allegretta ${ }^{7}$. Beatrice Detti ${ }^{1}$. Isacco Desideri ${ }^{6} \cdot$ Rolando Maria D'Angelillo $^{8}$. Laura Masi ${ }^{9}$. \\ Gianluca Ingrosso ${ }^{10} \cdot$ Mario Di Staso $^{11} \cdot$ Ercole Mazzeo $^{12} \cdot$ Fabio Trippa $^{13} \cdot$ Frank Lohr $^{12} \cdot$ Alessio Bruni $^{12}$. \\ Lorenzo Livi ${ }^{6}$
}

Received: 12 August 2021 / Accepted: 1 February 2022 / Published online: 5 March 2022

(c) Italian Society of Medical Radiology 2022

\begin{abstract}
Purpose To assess outcomes between salvage radiation therapy (SRT) with curative intent and stereotactic radiotherapy for macroscopic prostate recurrence (SSRT) after radical prostatectomy (RP). In order to compare these two different options, we compared their outcomes with a propensity score-based matched analysis.

Methods Data from 185 patients in seven Italian centres treated for macroscopic prostate bed recurrence after RP were retrospectively collected. To make a comparison between the two treatment groups, propensity matching was applied to create comparable cohorts.

Results After matching, 90 patients in the SRT and SSRT groups were selected (45 in each arm). Kaplan-Meier analysis did not show any significant differences in terms of BRFS and PFS between matched populations $(p=0.08$ and $p=0.8$, respectively). Multivariate models show that treatment was not associated with BRFS, neither in the whole or matched cohort, with HR of 2.15 (95\% CI $0.63-7.25, p=0.21)$ and 2.65 (95\% CI 0.59-11.97, $p=0.21$ ), respectively. In the matched cohort, lower rate of toxicity was confirmed for patients undergoing SSRT, with acute GI and GU adverse events reported in 4.4 versus $44.4 \%(p<0.001)$ and 28.9 versus $46.7 \%(p=0.08)$ of patients, and late GI and GU adverse events reported in 0 versus $13.3 \%(p=0.04)$ and 6.7 versus $22.2 \%(p=0.03)$ of patients, respectively.

Conclusion Considering the favourable therapeutic ratio of this approach and the lower number of fractions needed, SSRT should be considered as an attractive alternative to conventional SRT in this setting.
\end{abstract}

Keywords Prostate cancer $\cdot$ Macroscopic bed recurrence $\cdot$ Stereotactic radiotherapy $\cdot$ Salvage radiation therapy $\cdot$ Propensity score-based matched analysis

\section{Purpose/objective}

Radical prostatectomy (RP) represents one of the cornerstones for treatment of localized prostate cancer [1,2]. Nevertheless, nearly $30 \%$ of patients relapses after surgery and routinely undergo salvage radiation therapy (SRT) with curative intent [3]. However, patients with macroscopic local recurrence detected with positive metabolic imaging

Author Sara Gandini is responsible for statistical analyses.

Giulio Francolini

francolinigiulio@gmail.com

Extended author information available on the last page of the article commonly showed poor response to conventional SRT [4] and may need treatment intensification. Moreover, failure after SRT occurs in $82 \%$ of patients with a pre-SRT value $\geq 1.5 \mathrm{ng} / \mathrm{ml}$ [5-8]. Thus, local burden of disease negatively affects prognosis of these patients, and therapeutic ratio of conventional SRT may be further worsened by a rate of late grade $\geq 2$ GI and GU adverse events reported in the literature, ranging between 3.4 and 18\% [9-11]. In this setting, dose-escalated treatment may achieve better disease control [12]. However, poor outcomes in terms of disease control in this subgroup of patients characterized by macroscopic evidence of disease within prostate bed are an unmet clinical need. Strategies for treatment intensification exploiting all potential of modern radiotherapy 
techniques are eagerly awaited. Stereotactic techniques may offer acceptable disease control together with promising results in terms of toxicity, with an overall short treatment duration. Stereotactic radiotherapy for macroscopic prostate recurrence (SSRT), defined as hypofractionated RT ( $\geq 5$ Gy per fraction) with online image guidance, may be used to improve outcome in these patients, with potential advantages in terms of reduced treatment volumes, normal tissue injury and lower number of fractions. We recently published a multicentric retrospective series including 90 patients treated with this approach, reporting a rate of complete biochemical response (defined as a PSA nadir after treatment $\leq 0.2 \mathrm{ng} / \mathrm{ml}$ ) and biochemical relapse-free survival (BRFS) after 21.2 months of mean follow-up of $43.3 \%$ and $64.5 \%$, respectively [13]. To our knowledge, no direct comparisons between conventional prostate bed SRT and SSRT have been carried out, and retrospective case series could be prone to multiple confounding factors influencing oncological outcomes (e.g. baseline PSA at recurrence, etc.). In order to compare these two different options, we retrospectively collected data about patients with macroscopic prostate bed recurrence treated with these two approaches and compared their outcomes with a propensity score-based matched analysis.

\section{Material/methods}

\section{Population}

Data from 185 patients in seven Italian centres treated for macroscopic prostate bed recurrence after RP were retrospectively collected and reported. Overall, 90 and 95 patients underwent SSRT and SRT, respectively. Biochemical recurrence after RP was diagnosed according to European Association of Urology guidelines [14]. Staging was performed by physician choice, based on initial risk features and PSA at recurrence. Macroscopic evidence of local recurrence was obtained with magnetic resonance imaging (MRI), metabolic imaging such as choline or prostate-specific membrane antigen positron emission tomography-computed tomography (PSMA CT-PET), CT or/and biopsy. Patients with evidence of nodal or distant disease were excluded from the current analysis. The study was part of the retrospective research on prostate cancer notified to Ethical Committee (notification $\mathrm{Nr}$ 79). All patients included from the seven participating centres gave consent for the use of their anonymized data for research and educational purposes.

\section{Treatment}

Salvage radiotherapy was the only local treatment allowed, and the decision to perform SRT or SSRT was at the discretion of the treating radiation oncologist. No previous local treatment after recurrence was performed. Concomitant androgen deprivation therapy (ADT) was permitted. Patients in the SSRT group were treated with total doses of 30-40 Gy in five fractions, while patients in the SRT cohort were treated with a mean dose of 66-79 Gy in 33-38 fractions. SSRT was delivered either with a CyberKnife Robotic System $^{\mathrm{R}}$ (Accuray Inc., USA) or intensity-modulated radiotherapy (IMRT) performed through $\operatorname{Vero}^{\mathrm{R}}$ (BrainLab, D/ MHI, Japan). CyberKnife patients underwent radiopaque fiducials markers placement one week before planning CT scan [15]. MRI and/or CT-PET were co-registered with planning CT scan to help delineation of gross tumour volume (GTV), corresponding to macroscopic evidence of disease within the prostate bed. Clinical target volume (CTV) was obtained by adding a 2-mm margin to GTV. Bladder was excluded from target volume. Planning target volume consisted of a volumetric expansion of the CTV by $3 \mathrm{~mm}(1 \mathrm{~mm}$ in the posterior direction) and by $5 \mathrm{~mm}$ ( $3 \mathrm{~mm}$ in the posterior direction) for patients treated with CyberKnife ${ }^{R}$ and Vero $^{R}$, respectively. Patient alignment and intrafraction tracking were carried out by fiducial tracking for patients treated with CyberKnife ${ }^{R}$, and the InTempo ${ }^{R}$ System was used to alter imaging frequency between 15 and $60 \mathrm{~s}$ depending on the magnitude of prostatic bed motion detected. Daily cone-beam CT were performed to provide image guidance during VERO ${ }^{\mathrm{R}}$ IMRT treatment. Patients undergoing conventional SRT were treated both with 3D conformal or intensity-modulated technique. In SRT group, image guidance strategy was based on local practice and technique used. CTV consisted in standard postoperative contouring. Simultaneous integrated boost (SIB) using coregistered imaging was allowed. Whole pelvis irradiation was performed according to clinical choice. CTV-to-PTV margin was added according to local practice and image guidance performed. The following organs at risk (OARs) were contoured: rectum, bladder, bowel, urethra, penile bulb, femoral heads and bowel (Fig. 1).

\section{Outcome assessment}

After treatment, patients were followed with serum PSA and clinical assessment every three months. Genitourinary (GU) and gastrointestinal (GI) adverse events were recorded at each visit. Biochemical recurrence was defined as a PSA increase above $0.2 \mathrm{ng} / \mathrm{ml}$ for patients with a PSA nadir $<0.2 \mathrm{ng} / \mathrm{ml}$ or two consecutive PSA increases $>25 \%$ if compared to nadir in patients with a PSA nadir $>0.2 \mathrm{ng} / \mathrm{ml}$. Biochemical recurrence-free survival (BRFS) was defined as time from beginning of treatment to biochemical recurrence after SSRT or SRT, death or last follow-up. Progression-free survival (PFS) was defined as time from treatment start to any of the following, whichever occurred first: biochemical 

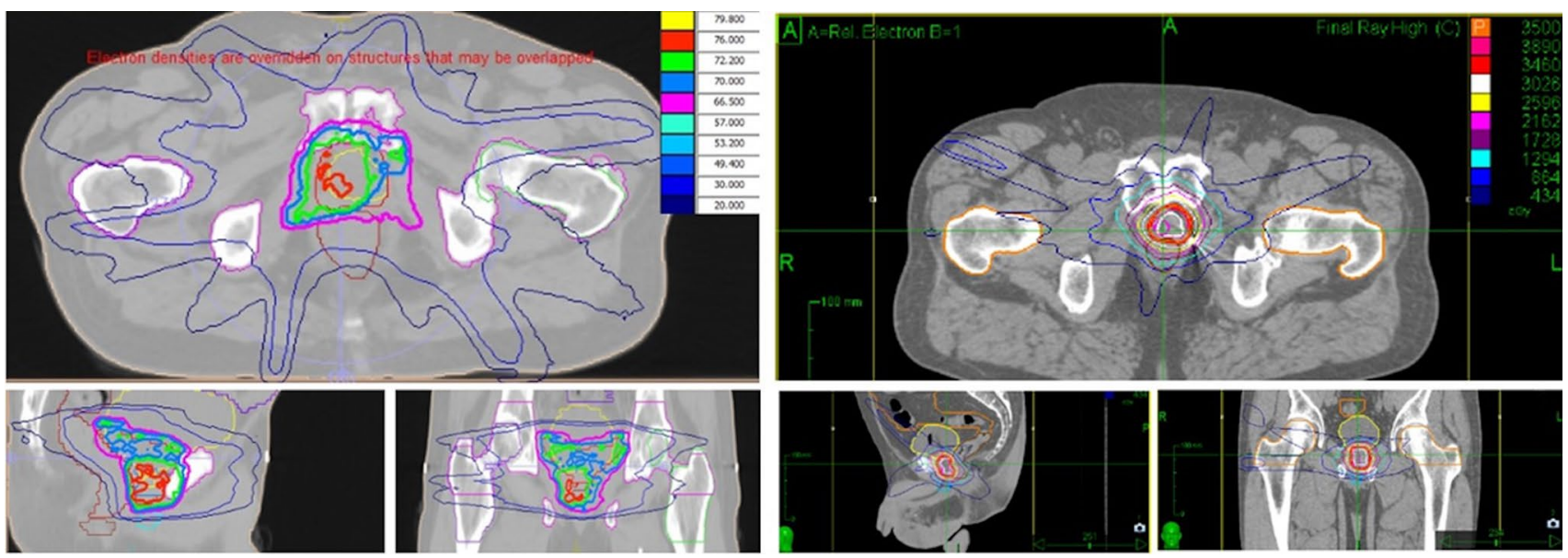

Fig. 1 Report snapshots of a SRT (left) versus a SSRT (right) sample plan with isodose lines

recurrence, locoregional or distant recurrence, death or last follow-up. Data about toxicity were collected from patient clinical records and reported according to Common Terminology Criteria for Adverse Events (CTCAE) score v.4.03 [16].

\section{Statistical analysis}

We used Chi-squared test, Fisher exact test and Wilcoxon rank test to assess differences in the distribution of categorical and continuous variables across groups of patients, respectively. In order to make a comparison between the two treatment groups, we calculated a propensity score using multivariable logistic models including factors that are widely reported to influence outcomes. Propensity scores reflect the probability that a patient received SSRT vs SRT based on baseline characteristics related to his prognostic factors. We presented the Odd Ratios (OR) and 95\% Confidence Intervals $(95 \% \mathrm{CI})$ for the associations between prognostic factors and confounding factors with RT treatment.

Propensity matching was applied to create comparable cohorts. Patients receiving SSRT were matched on a oneto-one basis with subjects receiving vs SRT. Matching was performed by using the R package "MatchIt" [17] based on nearest-neighbour matching. This matching procedure matches participants from the SSRT group to participants from the SRT group according to the absolute difference between their propensity scores which must result as the smallest to establish a match. Since the two treatment groups had significantly different median follow-up, events were censored at 2 years in order to make the two cohorts comparable. Statistical analyses were performed on the whole cohort, stratifying the analyses by risk strata identified with the propensity scores, and the subgroup of patients matched by propensity score. The first analysis can count on a big sample size that allows a greater statistical power, whereas the second has the advantage of two balanced cohorts in terms of prognostic and confounding factors.

Survival probabilities over time were estimated by the Kaplan-Meier method, and the univariate analyses to assess the differences between survival curves of different groups of patients were carried out by the log-rank test. To investigate the differences between treatments on time to relapse and OS, adjusting for age and stratifying for the propensity score, multivariate Cox proportional hazard models were performed and hazard ratios (HRs) with 95\% Confidence intervals $(95 \% \mathrm{CI})$ are presented.

Reported genitourinary (GU) and gastrointestinal (GI) toxicity, measured according to CTCAE score v.4.03, were compared with Chi-squared test or Fisher exact test. Multivariate logistic models were applied to investigate significant differences in acute toxicity between SSRT and SRT independently of significant confounders. We report Odd Ratios (ORs) and 95\% CI estimating the associations between acute toxicity and RT schedules.

In the analysis of the whole cohort we stratified for the propensity score, in the analysis on the matched subgroups, we adjusted only for confounders not included in the propensity score.

Statistical analyses were performed using the SAS statistical software (version 9.02 for Windows) and R (version 3.6.1).

\section{Results}

Data from clinical records of 185 patients consecutively treated for macroscopic prostate bed recurrence were retrospectively collected and reviewed. Ninety-five and 90 patients were treated with SRT and SSRT, respectively. 
Out of 185 patients included in the final analysis, 120 recurrences had been detected with choline CT-PET, five with PSMA CT-PET and two with both PSMA and Choline CT-PET. Pelvic MRI and CT were used to detect recurrence in 40 and six cases, respectively. Fourteen cases in the SRT group were confirmed with biopsy only. Median follow-up was 30 months in the entire cohort (IQR 16-56), while 18 months (IQR 9-30) and 55 months (IQR 33-100) were the median follow-up of the SSRT and SRT groups, respectively. Before matching, statistically significant difference between the two groups were found for age $(p<0.001)$, PSA at biochemical recurrence $(p<0.001)$ and concomitant ADT administration $(<0.001)$ (Table 1$)$. Median PSA at recurrence in the whole, unmatched cohort was $1.4 \mathrm{vs} 1.3 \mathrm{ng} / \mathrm{ml}$ in the SSRT vs RT group, respectively. After treatment, median PSA nadir was 0.04 vs $0.19 \mathrm{ng} / \mathrm{ml}$ in the SRT versus SSRT subgroup, respectively. After matching, 90 patients in the SRT and SSRT groups were selected (45 in each arm). Patients in the SSRT group had significantly higher median age and a higher rate of

Table 1 Cohort not matched

\begin{tabular}{|c|c|c|c|c|}
\hline & Total $(n=185)$ & $\operatorname{SSRT}(n=90)$ & SRT $(n=95)$ & $p$-value \\
\hline \multicolumn{5}{|c|}{ Age median (IQR) } \\
\hline & $62(58-71)$ & $71(66-76)$ & $58(54-61)$ & $<0.001$ \\
\hline \multicolumn{5}{|c|}{ Gleason score } \\
\hline$\leq 7$ & $147(79.46)$ & $66(73.33)$ & $81(85.26)$ & \multirow[t]{2}{*}{0.06} \\
\hline$>7$ & $38(20.54)$ & $24(26.67)$ & $14(14.74)$ & \\
\hline \multicolumn{5}{|c|}{ Pathological T } \\
\hline 2 & $110(59.46)$ & $46(51.11)$ & $64(67.37)$ & \multirow[t]{3}{*}{0.06} \\
\hline 3 & $68(36.76)$ & $39(43.33)$ & $29(30.53)$ & \\
\hline Unknown & $7(3.78)$ & $5(5.56)$ & $2(2.11)$ & \\
\hline \multicolumn{5}{|c|}{ Pathological N } \\
\hline 0 & $109(58.92)$ & $58(64.44)$ & $51(53.68)$ & \multirow[t]{4}{*}{0.22} \\
\hline 1 & $11(5.95)$ & $5(5.56)$ & $6(6.32)$ & \\
\hline $\mathrm{X}$ & $63(34.05)$ & $25(27.78)$ & $38(40)$ & \\
\hline Unknown & $2(1.08)$ & $2(2.22)$ & $0(0)$ & \\
\hline \multicolumn{5}{|c|}{ PSA pre-median (IQR) } \\
\hline & $8.8(6.1-12)$ & $8.3(5.9-12)$ & $9(6.7-12.7)$ & 0.86 \\
\hline \multicolumn{5}{|c|}{ Biochemical PSA median (IQR) } \\
\hline & $1.1(0.4-2.3)$ & $0.9(0.3-1.4)$ & $1.7(0.8-3.7)$ & $<0.001$ \\
\hline \multicolumn{5}{|c|}{ Time to relapse $<36$ months } \\
\hline Yes & $108(58.38)$ & $49(54.44)$ & $59(62.11)$ & \multirow[t]{2}{*}{0.36} \\
\hline No & 77 (41.62) & $41(45.56)$ & $36(37.89)$ & \\
\hline \multicolumn{5}{|c|}{ Clinical PSA median (IQR) } \\
\hline & $1.4(0.6-2.5)$ & $1.4(0.9-2.2)$ & $1.3(0.4-2.7)$ & 0.88 \\
\hline \multicolumn{5}{|c|}{ Hormonal therapy } \\
\hline Yes & $71(38.38)$ & $17(18.89)$ & $54(56.84)$ & \multirow[t]{2}{*}{$<0.001$} \\
\hline No & $114(61.62)$ & $73(81.11)$ & $41(43.16)$ & \\
\hline
\end{tabular}

Significant results are highlighted in bold

SSRT Stereotactic salvage radiation therapy, SRT Salvage radiation therapy concomitant ADT administration in the matched cohort ( $p<0.001$ for both) (Table 2). Kaplan-Meier analysis did not show any significant differences in terms of BRFS and PFS between matched populations ( $p=0.08$ and $p=0.8$, respectively) (Fig. 2). Multivariate models show that treatment was not significantly associated with BRFS, neither in the whole or matched cohort, with HR of 2.15 (95\% CI 0.63-7.25, $\mathrm{p}=0.21)$ and 2.65 (95\% CI 0.59-11.97, $p=0.21)$ in the whole and matched cohort, respectively (Table 3). Considering any grade toxicity in the whole unmatched cohort, rate of acute GI and GU toxicity was 3.3 versus $44.2 \%(p<0.001)$ and in 18.9 versus $39 \%$ $(p=0.002)$ in the SSRT vs SRT group, respectively. Rate of late GI and GU toxicity was 0 versus $11.6 \%(p=0.003)$ and in 3.3 vs $20 \%(p<0.001)$ in the SSRT vs SRT group, respectively. In the matched cohort, lower rate of toxicity was confirmed for patients undergoing SSRT, with acute GI and GU adverse events reported in 4.4 versus $44.4 \%$ $(p<0.001)$ and 28.9 versus $46.7 \%(p=0.08)$ of patients,

Table 2 Cohort matched

$\begin{array}{llll}\text { Total }(n=185) & \text { SSRT }(n=90) & \text { SRT }(n=95) & p \text {-value } \\ \text { TOT }(n=90) & \text { SSRT }(n=45) & \text { SRT }(n=45) & p \text {-value }\end{array}$

Age median (IQR)

$\begin{array}{llll}62(59-72) & 71(68-75) & 59(56-61) & <\mathbf{0 . 0 0 1}\end{array}$

$\leq 7 \quad 72(80)$

$>7 \quad 18(20)$

$37(82.22) \quad 35(77.78)$

0.79

Pathological T

$8(17.78) \quad 10(22.22)$

$57(63.33)$

$24(53.33)$

$33(73.33)$

0.13

$3 \quad 31(34.44) \quad 19(42.22) \quad 12(26.67)$

Unknown $2(2.22) \quad 2(4.44) \quad 0(0)$

Pathological $N$

\begin{tabular}{|c|c|c|c|c|}
\hline 0 & $50(55.56)$ & $27(60)$ & $23(51.11)$ & 0.63 \\
\hline 1 & $4(4.44)$ & $2(4.44)$ & $2(4.44)$ & \\
\hline$X$ & $35(38.89)$ & $15(33.33)$ & $20(44.44)$ & \\
\hline Unknown & $1(1.11)$ & $1(2.22)$ & $0(0)$ & \\
\hline \multicolumn{5}{|c|}{ PSA pre-median (IQR) } \\
\hline & $9(6.2-12)$ & $9(6.4-12.6)$ & $9(6.3-11.5)$ & 1 \\
\hline \multicolumn{5}{|c|}{ Biochemical PSA median (IQR) } \\
\hline & $1.14(0.4-2.1)$ & $1.03(0.4-1.8)$ & $1.25(0.4-2.3)$ & 0.67 \\
\hline \multicolumn{5}{|c|}{ Time to relapse $<36$ months } \\
\hline Yes & $52(57.78)$ & $26(57.78)$ & $26(57.78)$ & 0.36 \\
\hline No & $38(42.22)$ & $19(42.22)$ & $19(42.22)$ & \\
\hline \multicolumn{5}{|c|}{ Clinical PSA median (IQR) } \\
\hline & $1.14(0.6-2.3)$ & $1.16(0.7-1.9)$ & $1.1(0.4-2.3)$ & 1 \\
\hline \multicolumn{5}{|c|}{ Hormonal therapy } \\
\hline Yes & $32(35.56)$ & $25(55.56)$ & $7(15.56)$ & $<\mathbf{0 . 0 0}$ \\
\hline No & $58(64.44)$ & $20(44.44)$ & $38(84.44)$ & \\
\hline
\end{tabular}

Significant results are highlighted in bold

$S S R T$ Stereotactic salvage radiation therapy, SRT Salvage radiation therapy 
Kaplan Meier curves on matched cohort
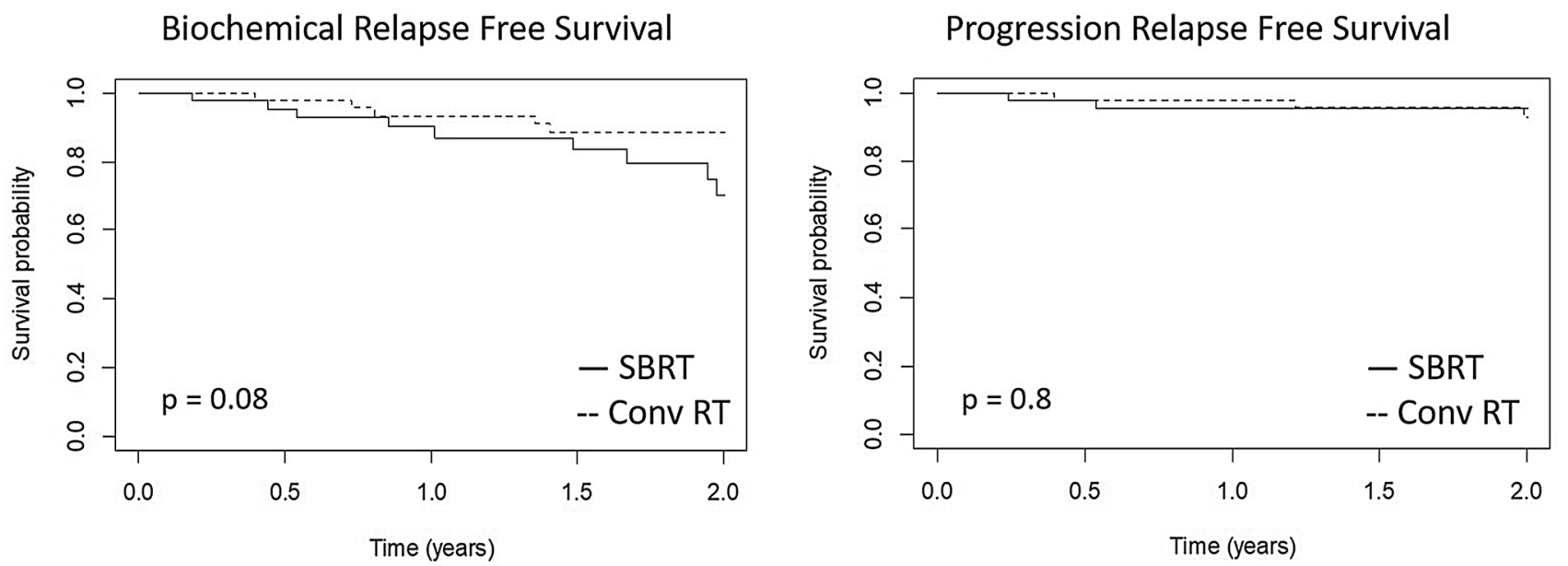

Fig. 2 Biochemical relapse-free survival and progression-free survival for stereotactic and conventional salvage radiotherapy

Table 3 Cox multivariate analysis results for unmatched and matched population

\begin{tabular}{|c|c|c|c|c|c|c|c|}
\hline \multirow[t]{2}{*}{ Variable } & \multirow[t]{2}{*}{ Contrast } & \multicolumn{3}{|c|}{ Unmatched } & \multicolumn{3}{|c|}{ Matched } \\
\hline & & HR & $95 \% \mathrm{CI}$ & $P$ & HR & $95 \% \mathrm{CI}$ & $p$ \\
\hline Treatment & SSRT versus SRT & 2.15 & $0.63-7.25$ & 0.21 & 2.65 & $0.59-11.97$ & 0.21 \\
\hline Age & & 1.04 & $0.98-1.09$ & 0.14 & 1.00 & $0.92-1.08$ & 0.94 \\
\hline
\end{tabular}

SSRT Stereotactic salvage radiation therapy, SRT Salvage radiation therapy and late GI and GU adverse events reported in 0 vs $13.3 \%$ $(p=0.04)$ and 6.7 versus $22.2 \%(p=0.03)$ of patients, respectively (Table 4 ). In the overall cohort, $\geq \mathrm{G} 2$ acute GI toxicity was reported in 0 and $18(17 \mathrm{G} 2,1 \mathrm{G} 3)$ patients in the SSRT and SRT group, while $\geq \mathrm{G} 2$ acute GU toxicity occurred in $1(\mathrm{G} 2)$ and $14(11 \mathrm{G} 2$ and $3 \mathrm{G} 3)$ patients in the SSRT and SRT group, respectively. Late GI $\geq \mathrm{G} 2$ toxicity was reported in 0 and 10 (all G2) patients in the SSRT and SRT group, while $\geq \mathrm{G} 2$ late GU toxicity occurred in 2 (all $\mathrm{G} 2)$ and 8 (7 G2 and $1 \mathrm{G} 3$ ) patients in the SSRT and SRT group, respectively. Considering matched cohort, $\geq \mathrm{G} 2$ acute GI toxicity was reported in 0 and 7 (all G2) patients in the SSRT and SRT group, while $\geq \mathrm{G} 2$ acute GU toxicity occurred in $1(\mathrm{G} 2)$ and 8 (7 G2 and $1 \mathrm{G} 3$ ) patients in the SSRT and SRT group, respectively. Late GI $\geq$ G2 toxicity was reported in 0 and 4 (all G2) patients in the SSRT and SRT group, while $\geq \mathrm{G} 2$ late GU toxicity occurred in 2 (all G2) and 4 (3 G2 and $1 \mathrm{G} 3$ ) patients in the SSRT and SRT group, respectively. After adjustment for treatment dose, treating centre was significantly related to acute GI adverse events $(p=0.0353)$ and late $\mathrm{GU}(p=0.0002)$ and GI toxicity $(p=0.0358)$ in the SRT group, while no significant influence of this factor was found in the SSRT group (Supplementary Table 1).

\section{Discussion}

Results from the present analysis suggest comparable oncologic outcome in the two groups with improved tolerability of SSRT. No difference was reported in terms of BRFS. Considering the favourable therapeutic ratio of this approach and the lower number of fractions needed to treat the patients, SSRT should be considered as an attractive alternative to conventional SRT in patients affected by macroscopic prostate bed recurrence after RP. Our previous experience already showed a promising biochemical control with significant ADT delay [13]. RTOG 9601 showed a biochemical disease control at 12 years after conventional SRT approach of $67.9 \%$ without ADT. However, $80.7 \%$ in the placebo arm of that trial had a PSA at enrolment $\leq 0.7 \mathrm{ng} / \mathrm{ml}$ [18], and a comparison could be difficult, considering adverse prognostic features in our series. In fact, $73.5 \%$ of patients included in the present analysis had a PSA $\geq 0.7 \mathrm{ng} / \mathrm{ml}$ at treatment, and macroscopic tissue within prostate bed was evident in all cases. A retrospective study including only patients with similar features undergoing SRT was published in 2019, showing overall survival and distant metastasis-free survival of 
Table 4 Toxicity cohort (matched and unmatched cohorts)

\begin{tabular}{|c|c|c|c|c|}
\hline & \multicolumn{4}{|l|}{ Matched cohort } \\
\hline & TOT $(\mathrm{n}=185)$ & $\operatorname{SSRT}(n=90)$ & SRT $(n=95)$ & $p$-value \\
\hline \multicolumn{5}{|c|}{ GI acute toxicity } \\
\hline Yes & $45(24.32 \%)$ & $3(3.33 \%)$ & $42(44.21 \%)$ & \multirow[t]{2}{*}{$<0.001$} \\
\hline No & $140(75.68 \%)$ & $87(96.67 \%)$ & $53(55.79 \%)$ & \\
\hline \multicolumn{5}{|c|}{ GU acute toxicity } \\
\hline Yes & $54(29.19 \%)$ & $17(18.89 \%)$ & $37(38.95 \%)$ & \multirow[t]{2}{*}{0.002} \\
\hline No & $131(70.81 \%)$ & $73(81.11 \%)$ & $58(61.05 \%)$ & \\
\hline \multicolumn{5}{|c|}{ GI late toxicity } \\
\hline Yes & $11(5.95 \%)$ & $0(0 \%)$ & $11(11.58 \%)$ & \multirow[t]{2}{*}{0.003} \\
\hline No & $174(94.05 \%)$ & $90(100 \%)$ & $84(88.42 \%)$ & \\
\hline \multicolumn{5}{|c|}{ GU late toxicity } \\
\hline Yes & $22(11.89 \%)$ & $3(3.33 \%)$ & $19(20 \%)$ & \multirow[t]{2}{*}{$<0.001$} \\
\hline \multirow[t]{3}{*}{ No } & $163(88.11 \%)$ & $87(96.67 \%)$ & $76(80 \%)$ & \\
\hline & \multicolumn{4}{|c|}{ Unmatched cohort } \\
\hline & TOT $(n=90)$ & SSRT $(n=45)$ & $\operatorname{SRT}(n=45)$ & $p$-value \\
\hline \multicolumn{5}{|c|}{ GI acute toxicity } \\
\hline Yes & $22(24.44 \%)$ & $2(4.44 \%)$ & $20(44.44 \%)$ & \multirow[t]{2}{*}{$<0.001$} \\
\hline No & $68(75.56 \%)$ & $43(95.56 \%)$ & $25(55.56 \%)$ & \\
\hline \multicolumn{5}{|c|}{ GU acute toxicity } \\
\hline Yes & $34(37.78 \%)$ & $13(28.89 \%)$ & $21(46.67 \%)$ & \multirow[t]{2}{*}{0.08} \\
\hline No & $56(62.22 \%)$ & $32(71.11 \%)$ & $24(53.33 \%)$ & \\
\hline \multicolumn{5}{|c|}{ GI late toxicity } \\
\hline Yes & $7(7.78 \%)$ & $0(0 \%)$ & $6(13.33 \%)$ & \multirow[t]{2}{*}{0.04} \\
\hline No & $83(92.22 \%)$ & $45(100 \%)$ & $39(86.67 \%)$ & \\
\hline \multicolumn{5}{|c|}{ GU late toxicity } \\
\hline Yes & $13(14.44 \%)$ & $3(6.67 \%)$ & $10(22.22 \%)$ & \multirow[t]{2}{*}{0.03} \\
\hline No & $77(85.56 \%)$ & $42(93.33 \%)$ & $35(77.78 \%)$ & \\
\hline
\end{tabular}

SSRT Stereotactic salvage radiation therapy, SRT Salvage radiation therapy, GI Gastro intestinal, $G U$ Genitourinary

76.1 and $73.3 \%$ at 10 years, respectively [12]. Populations with these baseline features are atypical, because early SRT within a PSA range between 0.2 and $0.5 \mathrm{ng} / \mathrm{ml}$ should be the standard approach [8]. Thus, a propensity scorebased matched analysis comparing two different salvage approaches in this setting could be useful to decrease the impact of confounding factors on outcomes despite the nonrandomized design. Potential limitations of the current series are related to its retrospective nature, the short follow-up and the non-homogenous sample treated with different techniques and dose-volume schedules. In particular, one of the potential issues of the present study is the difference in terms of treated volumes between the two groups. Indeed, 23 out of 95 patients in the SRT cohort were treated with whole pelvis prophylactic irradiation, potentially influencing the rate of adverse events in this cohort. However, only four patients treated to the whole pelvis were included in the matched population, reducing the impact of this confounder in these patients. Another issue could be represented by the subgroup of patients included in the SRT group staged through biopsy only. Nonetheless, this cohort is limited to 12 cases only, out of whom only two were included in the final matched analysis, limiting its potential impact on final results. SRT was administered with different techniques, including both 3D-CRT and IMRT, with a potential impact on longterm toxicity in this subgroup. However, this represents a realistic representation of current clinical practice across different facilities for SRT. Furthermore, long-term benefit of new technologies in this setting is unclear according to a large SEER database analysis [19], reducing the potential bias related to treatment heterogeneity in this arm. Impact of treating institution in terms of reported toxicities could be explained by technical difference of SRT administration. Indeed, patients treated in institutions routinely administering SIB were more prone to late GI and GU toxicities, suggesting that this technique may be related to increased toxicity. Of note, impact of 
treating institution was not significant in SSRT group. Of course, SSRT requires adequate technology and must be performed in centres with proven experience in this setting. Rate of patients undergoing concomitant ADT and SSRT was significantly higher in the matched population, with a potential influence on oncological results. However, benefit of concomitant ADT in this setting is still debated [20], and its impact on patients undergoing salvage treatment may be less than expected, especially in a population in which regional and distant disease was carefully excluded through pre-treatment imaging. Nonetheless, a longer follow-up would be needed to strengthen these results. Furthermore, substantial equivalence in terms of treatment outcome despite higher rate of concomitant ADT may be related to low isoeffective dose (EQD2) in the SSRT arm. Whether an increased biochemical control could be obtained through further dose escalation in an interesting issue. Sampath et al. recently tested stereotactic dose-escalated radiotherapy on prostate bed in a prospective phase I study, showing a crude rate of biochemical control of $42 \%$ in the overall population. Patients were treated with dose fractionation schedules of 35,40 and $45 \mathrm{~Gy}$ in five fractions. Authors underlined that dose escalation to $45 \mathrm{~Gy}$ was feasible without increasing the rate of adverse events, but no improvement in PSA control was reported if compared to $40 \mathrm{~Gy}$ in 5 fractions [21]. Of note, macroscopic evidence of tumour tissue within prostate bed could occur more often in the near future after clinical implementation of new imaging modalities. For example, PSMA PET/CT has demonstrated promising results for re-staging after RP [22], with reported detection rates of $78-81 \%$ [23, 24]. Whether a PSMA-guided approach consisting in early SSRT on prostate bed recurrence could be cost-effective is intriguing and should be object of prospective evaluation.

\section{Conclusion}

Significantly lower toxicity rate was observed after SSRT while maintaining similar oncological outcomes, when compared to SRT. These findings seem to suggest that SSRT may be considered a valid alternative for post-prostatectomy macroscopic local recurrence, offering short (convenient), cost-effective, well-tolerated and efficacious approach. Interestingly, shorter treatment schedule may be particularly useful during the COVID-19 pandemic [25, 26]. Moreover, SSRT may offer a valid approach to implement information offered by new imaging modalities (e.g. PSMA PET/CT) in postoperative management of prostate cancer relapses. Dose escalation protocols (e.g. $40 \mathrm{~Gy}$ in five fractions) may further enhance therapeutic ratio of this approach and exploit all its potential benefit. These results could prompt significant shifts in routine postoperative management of prostate cancer [27] and use of stereotactic radiotherapy for oligorecurrent disease [28]. Even if these results seem to be very promising, longer follow-up is needed to better understand the improvement showed by SSRT in terms of acute/ late side effects and clinical outcomes.

Supplementary Information The online version contains supplementary material available at https://doi.org/10.1007/s11547-022-01465-w.

Funding BA Jereczek-Fossa has received research support from Accuray (institutional grant), AIRC Italian Association for Cancer Research (grants). BA Jereczek-Fossa has received travel support or honoraria from Janssen, Ferring, Bayer, Roche, Astellas, Elekta, Carl Zeiss and Ipsen.

\section{Declarations}

Conflict of interest On behalf of all authors, the corresponding author states that there is no conflict of interest.

Ethical standards This article does not contain any studies with human participants or animals performed by any of the authors.

\section{References}

1. Tyson MD 2nd, Andrews PE, Ferrigni RF et al (2016) Radical prostatectomy trends in the United States: 1998 to 2011. Mayo Clin Proc. 91(1):10-6

2. Buglione M, Noale M, Bruni A et al (2019) Treatment paths for localised prostate cancer in Italy: the results of a multidisciplinary, observational, prospective study (Pros-IT CNR). PLoS One 14(11):e0224151

3. Thompson IM, Valicenti RK, Albertsen P et al (2013) Adjuvant and salvage radiotherapy after prostatectomy: AUA/ASTRO Guideline. J Urol 190:441-449

4. Emmett L, Van Leeuwen PJ, Nandurkar R et al (2017) Treatment outcomes from 68Ga-PSMA PET/CT-informed salvage radiation treatment in men with rising psa after radical prostatectomy: prognostic value of a negative PSMA PET. J Nucl Med. 58(12):1972-1976

5. Stephenson AJ, Slawin KM, Bianco FJ Jr et al (2004) Perspectives on the natural history of recurrent prostate cancer after radical prostatectomy, based on the response to salvage radiotherapy. BJU Int 94:1210-1212

6. Carrie C, Hasbini A, de Laroche G et al (2016) Salvage radiotherapy with or without short-term hormone therapy for rising prostate-specific antigen concentration. after radical prostatectomy (GETUG-AFU 16): a randomised, multicentre, open-label phase 3 trial. Lancet Oncol 17:747-756

7. Tendulkar RD, Agrawal S, Gao T et al (2016) Contemporary update of a multi-institutional predictive nomogram for salvage radiotherapy after radical prostatectomy. J Clin Oncol 34(30):3648-3654

8. Stephenson AJ, Scardino PT, Kattan MW et al (2007) Predicting the outcome of salvage radiation therapy for recurrent prostate cancer after radical prostatectomy. J Clin Oncol 25:2035-2041 
9. Ingrosso G, Carosi A, di Cristino D et al (2017) Volumetric image-guided highly conformal radiotherapy of the prostate bed: toxicity analysis. Rep Pract Oncol Radiother Jan-Feb 22(1):64-70

10. Nath SK, Sandhu AP, Rose BS et al (2010) Toxicity analysis of postoperative image-guided intensity-modulated radiotherapy for prostate cancer. Int J Radiat Oncol Biol Phys 78(2):435-441

11. Bellavita R, Massetti M, Abraha I et al (2012) Conformal postoperative radiotherapy in patients with positive resection margins and/or pT3-4 prostate adenocarcinoma. Int J Radiat Oncol Biol Phys 84(3):e299-e304

12. Bruni A, Ingrosso G, Trippa F et al (2019) Macroscopic locoregional relapse from prostate cancer: which role for salvage radiotherapy? Clin Transl Oncol. https://doi.org/10.1007/ s12094-019-02084-0

13. Francolini G, Jereczek-Fossa BA, Di Cataldo V, Simontacchi G, Marvaso G, Zerella MA, Gentile P, Bianciardi F, Allegretta S, Detti B, Masi L, Lo Russo M, Livi L (2020) Stereotactic radiotherapy for prostate bed recurrence after prostatectomy, a multicentric series. BJU Int 125(3):417-425

14. Cornford P, Bellmunt J, Bolla M et al (2017) EAU-ESTROSIOG guidelines on prostate cancer: part II: treatment of relapsing, metastatic, and castration-resistant prostate cancer. Eur Urol. 71(4):630-642

15. De Cicco L, Bracelli S (2019) Fiducial markers implantation for prostate image-guided radiotherapy: a report on the transperineal approach. Radiol Med 124(2):132-135

16. U.S. Department of Health and Human Services NIH, National Cancer Institute. Common Terminology Criteria for Adverse Events (CTCAE) Version 4.0. https://evs.nci.nih.gov/ftp1/ CTCAE/CTCAE_4.03/CTCAE_4.03_2010-0614_QuickRefer ence_5x7.pdf. Last accessed February, 272020

17. Ho D, Imai K, King G et al (2011) MatchIt: nonparametric preprocessing for parametric causal inference. J Stat Softw 42(8): $1-28$

18. Shipley WU, Seiferheld W, Lukka HR et al (2017) NRG oncology RTOG: Radiation With Or Without Antiandrogen Therapy In Recurrent Prostate Cancer. N Engl J Med. 376(5):417-428

19. Goldin GH, Sheets NC, Meyer AM et al (2013) Comparative effectiveness of intensity-modulated radiotherapy and conventional conformal radiotherapy in the treatment of prostate cancer after radical prostatectomy. JAMA Intern Med 173(12):1136-1143

20. Spratt DE (2018) Evidence-based risk stratification to guide hormone therapy use with salvage radiation therapy for prostate cancer. Int J Radiat Oncol Biol Phys 102(3):556-560

21. Sampath S, Frankel P, Vecchio BD et al (2019) Stereotactic body radiation therapy to the prostate bed: results of a phase 1 doseescalation trial. Int J Radiat Oncol Biol Phys. 106:537

22. Afshar-Oromieh A, Zechmann CM, Malcher A et al (2014) Comparison of PET imaging with a (68)Ga-labelled PSMA ligand and
(18)F-choline-based PET/CT for the diagnosis of recurrent prostate cancer. Eur J Nucl Med Mol Imaging 41(1):11-20. https:// doi.org/10.1007/s00259-013-2525-5

23. Perera M, Papa N, Roberts M et al (2019) Gallium-68 prostatespecific membrane antigen positron emission tomography in advanced prostate cancer-updated diagnostic utility, sensitivity, specificity, and distribution of prostate-specific membrane antigen-avid lesions: a systematic review and meta-analysis. Eur Urol 77:403

24. von Eyben FE, Picchio M, von Eyben R et al (2018) 68Ga-labeled prostate-specific membrane antigen ligand positron emission tomography/computed tomography for prostate cancer: a systematic review and meta-analysis. Eur Urol Focus 4:686-693

25. Barra S, Guarnieri A, di Monale E, Bastia MB, Marcenaro M, Tornari E, Belgioia L, Magrini SM, Ricardi U, Corvò R (2021) Short fractionation radiotherapy for early prostate cancer in the time of COVID-19: long-term excellent outcomes from a multicenter Italian trial suggest a larger adoption in clinical practice. Radiol Med 126(1):142-146

26. Francolini G, Desideri I, Stocchi G, Ciccone LP, Salvestrini V, Garlatti P, Aquilano M, Greto D, Bonomo P, Meattini I, Scotti V, Scoccianti S, Simontacchi G, Livi L (2021) Impact of COVID-19 on workload burden of a complex radiotherapy facility. Radiol Med 126(5):717-721

27. Alongi F, De Bari B, Franco P, Ciammella P, Chekrine T, Livi L, Jereczek-Fossa BA, Filippi AR, AIRO Young and AIRO Prostate cancer Working Group (2013) The PROCAINA (PROstate CAncer INdication Attitudes) project (Part I): a survey among Italian radiation oncologists on postoperative radiotherapy in prostate cancer. Radiol Med 118(4):660-678

28. Jereczek-Fossa BA, Bortolato B, Gerardi MA, Dicuonzo S, Arienti VM, Berlinghieri S, Bracelli S, Buglione M, Caputo M, Catalano G, Cazzaniga LF, De Cicco L, Di Muzio N, Filippone FR, Fodor A, Franceschini D, Frata P, Gottardo S, Ivaldi GB, Laudati A, Magrini SM, Mantero E, Meaglia I, Morlino S, Palazzi M, Piccoli F, Romanelli P, Scorsetti M, Serafini F, Scandolaro L, Valdagni R, Orecchia R, Antognoni P, Lombardy Section of the Italian Society of Oncological Radiotherapy (Associazione Italiana di Radioterapia Oncologica-Lombardia, AIRO-L) (2019) Radiotherapy for oligometastatic cancer: a survey among radiation oncologists of Lombardy (AIRO-Lombardy), Italy. Radiol Med. 124(4):315-322

Publisher's Note Springer Nature remains neutral with regard to jurisdictional claims in published maps and institutional affiliations.

\section{Authors and Affiliations}

\section{Giulio Francolini ${ }^{1}$ (D) Barbara Alicja Jereczek-Fossa ${ }^{2,3} \cdot$ Vanessa Di Cataldo ${ }^{1,4} \cdot$ Gabriele Simontacchi $^{1}$. Giulia Marvaso $^{2,3}$ - Sara Gandini ${ }^{5}$. Federica Corso ${ }^{5} \cdot$ Lucia Pia Ciccone $^{6} \cdot$ Maria Alessia Zerella $^{2,3} \cdot$ Piercarlo Gentile $^{7}$. Federico Bianciardi ${ }^{7}$. Sara Allegretta ${ }^{7}$. Beatrice Detti ${ }^{1} \cdot$ Isacco Desideri $^{6} \cdot$ Rolando Maria D'Angelillo $^{8}$. Laura Masi $^{9}$. Gianluca Ingrosso $^{10} \cdot$ Mario Di Staso $^{11} \cdot$ Ercole Mazzeo $^{12}$. Fabio Trippa ${ }^{13} \cdot$ Frank Lohr $^{12} \cdot$ Alessio Bruni $^{12}$. Lorenzo Livi ${ }^{6}$}

Sara Gandini

sara.gandini@ieo.it

1 Radiation Oncology Unit, Azienda OspedalieroUniversitaria Careggi, Viale Morgagni 85, 50134 Florence, Italy
2 Department of Oncology and Hemato-Oncology, University of Milan, Milan, Italy

3 Division of Radiotherapy, IEO European Institute of Oncology, IRCCS, Milan, Italy 
4 CyberKnife Center, Istituto Fiorentino di Cura ed Assistenza, Florence, Italy

5 Department of Experimental Oncology, European Institute of Oncology IRCCS, Via Adamello 16, 20139 Milan, Italy

6 Department of Biomedical, Experimental, and Clinical Sciences "Mario Serio", University of Florence, Florence, Italy

7 UPMC San Pietro FBF, Rome, Italy

8 Department of Radiation Oncology, Policlinico Tor Vergata University, Viale Oxford 81, 00133 Rome, RM, Italy

9 Department of Medical Physics and Radiation Oncology, IFCA, 50139 Florence, Italy
10 Radiation Oncology Section, Department of Medicine and Surgery, Perugia General Hospital, University of Perugia, Perugia, Italy

11 Radiotherapy Unit, "Nuovo San Salvatore” Hospital, L'Aquila, Italy

12 Radiotherapy Unit, University Hospital of Modena, Modena, Italy

13 Radiotherapy Oncology Centre, Azienda Ospedaliera S. Maria, Terni, Italy 